\title{
7 The datafication of therapeutic life management
}

\section{Assembling the self in control society}

\author{
Harley Bergroth and Ilpo Helén
}

For people who are willing or obliged to reflect on and proactively modify their personal conduct, a plethora of self-tracking devices are now widely available. By self-tracking devices we refer to near-body gadgets and related software applications that provide measurements of the rhythms and patterns of everyday life - for example, step counts, heart rate, walking distances and sleeping patterns. By providing quantitative data about vital functions or behavioural patterns, these technologies aim at helping people to enhance their self-knowledge, to adjust their behaviour and/or to accomplish self-improvement. As such, self-tracking technologies are entering and altering the domain of the 'therapeutic' that emerged and was consolidated during the 20th century (see Madsen, 2014, 2015; Moskowitz, 2001). Instead of approaching self-tracking as merely an instantiation of an overarching and static 'therapy culture', in this chapter we study more closely the therapeutic imaginaries and functions of self-tracking in everyday life, and situate the phenomenon as part of always-emergent therapeutic assemblages.

Self-tracking has emerged in conjunction with sociotechnical trajectories that are characterised by the terms 'data-driven' and 'datafication' in recent discussions (see Pentland, 2013; Ruckenstein \& Schüll, 2017). The terms 'data-driven' and 'datafication' refer to the collection and mining of masses of digital data by high-performance computers. Such practices are expanding to all walks of life, from healthcare to traffic and from energy production to mass entertainment. Advocates of data-driven technologies attach great expectations to their capability to provide precise and predictive control and steering of complex technical systems, including social organisations as well as individual human lives and behaviour (e.g. Mayer-Schönberger \& Cukier, 2013; Pentland, 2013; Topol, 2012, 2015). Self-tracking is undoubtedly an important dimension of this development, as it is essentially about the collection and analysis of cumulative data on bodies and personal lives. As self-tracking technologies increasingly saturate wellbeing-related retail contexts and cultural imaginaries of self-care, self-tracking can well be conceived of as a mode of datafication of everyday life (Lupton, 2016; Ruckenstein \& Schüll, 2017).

In order to grasp the formation of therapeutic regimes of action within this data-driven practice, we approach self-tracking and its therapeutic functions with the help of two concepts. First, we deploy the idea of assemblage (agencement) 
(Deleuze \& Guattari, 1987; Marcus \& Saka, 2006), which enables an analysis of self-tracking as a mode of action taking shape within and through networks and collections of things and discourses. We focus on how bodies, (technological) objects and political ideas of self-development and self-care come together in self-tracking and form therapeutic assemblages that 'hang together' (Mol, 2002) by and through the practice of gathering and analysing data about oneself. Furthermore, the concept of assemblage entails that the assemblage as a whole as well as its parts are in a constant state of emergence (Bennett, 2010; Latour, 2005). This enables us to situate the phenomenon of self-tracking within a global therapeutic assemblage while being sensitive to the possibilities, affordances and relations through which the idea of the 'therapeutic' itself becomes a meaningful concept in and through these practices. Second, we lean on Gilles Deleuze's (1995a, 1995b) ideas of control society and, more specifically, of 'dividualisation', which are particularly relevant as regards the therapeutic and political functioning of self-tracking. The concept of the dividual enables us to focus on the data-driven character of self-tracking, that is, how the practice of self-tracking becomes functional by and through data. It also helps us to situate self-tracking in current biopolitical assemblages in which individuals, their lives and their experienced selves increasingly become reduced to, as well as lived by and through, quantification-based haptic and visual information.

Drawing from qualitative analysis of promotional Internet material and interviews with Finnish self-trackers, we focus on the questions of how self-tracking becomes a therapeutic practice and what the idea of the 'therapeutic' might mean in the context of such a data-driven practice. We also ask how self-tracking, with its data-driven character, possibly shapes our understanding of the therapeutic. By studying self-tracking practices in this way, we are able to pinpoint contradictions that problematise a straightforward relationship between datafied life management and the therapeutic ethos.

This chapter proceeds as follows. First, we consider self-tracking in relation to the current therapeutic ethos and as an embodiment of dividualisation (Deleuze, 1995b). Then we present our data and methods. After this, we commence our analysis; first, we focus on the framing of self-tracking as a holistic therapeutic practice by technology developers, and show how self-tracking is reflective of what we call fragmentary holism. Secondly, we draw on interviews with Finnish self-trackers to elaborate on how self-tracking becomes a therapeutic practice in action and promotes regimes of perpetual self-assembly. Finally, we discuss briefly the political dimensions of self-tracking as self-control, and provide some concluding remarks.

\section{Proactive self-tracking and the therapeutic ethos}

People have used analogue technologies for self-measurement and lifelogging (Crawford et al., 2015; Lupton, 2016), for various reasons, for centuries. However, it may be argued that the rise of a 'therapy culture' - the relatively recent success of various forms of therapeutic life-management products and services which often 
involve some sort of reflective tracking practices (see McGee, 2005; Madsen, 2015) - has also driven the design, marketing and hype around contemporary digital self-tracking technologies. Whereas such technologies originally occupied quite specific domains such as (clinical) healthcare and competitive sports, ${ }^{1}$ the therapeutic idea of pursuing a better life through holistic self-improvement combined with the cultural power of metrics and data (Ruckenstein, 2014: 77; Beer, 2016) has paved the way for the adoption of quantification-driven self-tracking technologies in various spheres of everyday life. Today, consumers certainly find these technologies an integral part of wellness-related retail contexts, and digital self-tracking has consolidated its position as a mundane way of making sense of and possibly transforming - the body and the self.

Although the variety of regimens of self-help and self-improvement is vast in Western therapy culture (Illouz, 2008; Madsen, 2015), it is quite common in the therapeutic landscape to see that such therapeutic hermeneutics of the self (Foucault, 1993) take place in a reflexive dialogue. In it, a therapeutic instance - a psychotherapist, spiritual guide, peer group, self-help manual or a website - functions as a mirror or an echo that enables the person to acquire insights about who $\mathrm{s} /$ he actually is and what $\mathrm{s} / \mathrm{he}$ can become. It is tempting to think of self-tracking technologies as such mirrors since self-tracking may be thought of as a practice of negotiation with 'data doubles' (Ruckenstein, 2014; Lupton, 2013; also Lomborg \& Frandsen, 2016) that serve as a basis for investigating one's own life. Sociologist Deborah Lupton $(2013,2016$ : 39) associates self-tracking more clearly with contemporary self-help, as she links the proliferation of self-monitoring practices to the rise of popular psychology discourses that offer individualised solutions to personal problems. She analyses self-tracking as self-help-like responsibilisation of citizens on their own health and well-being. Lupton's arguments are congruent with critical studies that approach the Anglo-American therapeutic self-help as a field of neoliberal self-optimisation and governing. Such critical studies often see therapeutic self-help as a depoliticizing force as it may effectively hide structural and political problems by endorsing the management of personal qualities, traits or personality (e.g. Rimke, 2000; McGee, 2005; Madsen, 2015). However, the idea of an assemblage helps us to see that while self-tracking as a field of action is no doubt influenced and shaped by other forms of therapy culture, a focus on how the 'self' becomes enacted in practice within everyday self-tracking assemblages reveals a logic of dividualisation rather than that of individualisation. We argue that self-tracking reflects the interplay of the therapeutic ethos and contemporary, dividualising mechanisms of control, which also inverts the focus on the self/ person as a coherent whole found in much 20th-century self-help.

Dividualisation is a term employed by the French philosopher Gilles Deleuze (1995a, 1995b). With an eye on the proliferation of information technologies at the dawn of the digital Internet age in the 1990s, he presented an outline of control society. He claimed that corrective and normalising interventions by public authorities and experts are increasingly being replaced by practices of control that divide its objects into ever smaller elements, parameters and action-units. Deleuze considered dividualisation as a basic requirement of the functioning of control 
in 'societies that no longer operate by confining people but through continuous control and instant communication' (Deleuze, 1995a: 174). The rise of digital systems would bring about practices and technologies of monitoring and modulation that work within the flows and transactions between the forces and capacities of living human beings, the environments they live in and the practices in which they participate. For Deleuze, control is continuous and anticipatory, and it works with the help of predictive and prognostic information. The objects of control are conceived of as 'factors' or 'variables', and their interaction and conjugations are assessable as 'risks' and open to modification by meticulous operations and interventions. As a consequence, living human beings as communities, persons and organisms - as units typically conceived of as individuals - as well as the environments they live in, become transformed and fragmented into dividuals within the matrices of control. In short, dividualization refers to the construction of clear-cut variables, dividing 'everything' - vital functions, life events, mood changes, the person, etc. - according to these variables, gathering data about these variables, and looking for patterns, trends or deviations by aggregating the data. Dividualisation pertains to all levels of human existence: to populations as well as to basic biological processes on the cellular and molecular level. It is in connection to dividualisation that we begin to see the logic of holistic self-understanding or holistic transformation, often emphasised in therapeutic parlance, giving way to a more fragmentary understanding of therapeutic life-management.

\section{Data and methods}

Our analysis is based on a variety of qualitative research material. In order to examine how self-tracking is presented within the cultural imaginary, we collected textual material from webpages of individuals and organisations that promote and endorse everyday self-tracking. These materials include Quantified Self-related website publications, public blog posts as well as promotional materials of private enterprises that manufacture self-tracking devices. Furthermore, to grasp the experiences related to the therapeutic regimes of action in everyday self-tracking practices, we employed 15 semi-structured interviews conducted with Finnish self-trackers. The interviews were gathered during 2015-2016. Of the interviewees, six were men and nine were women, all of them were employed, studying or both at the time of the interviews. During the last few years, the first author has engaged in diverse self-tracking practices, including an eight-month period of consistently wearing a popular, consumer-grade activity tracking wristband (FitBit Charge HR). This, we feel, has enabled tacit knowledge on the studied field and the 'workings' of personal self-tracking assemblages in everyday life.

In order to investigate our overarching research questions, we conducted a close reading of our research material through two main themes. First, we looked into how self-tracking technologies and the data they produce are presented as being useful and meaningful for people in self-discovery, self-adjustment and self-improvement. Second, we investigated how the data serve - and how they are narrated as serving - the pursuit of a good life. Our analysis is not focused on 
human-device relations, and we do not try to individuate patterns of use for the deployment of self-tracking devices. Instead, we study self-tracking as a practice and technology of the self (Foucault, 1988). This means that we have analysed how self-tracking as a regime of action springs from, mediates and shapes one's relation to the self, and how the self becomes examined, understood and enacted through self-tracking. In addition, the concept of assemblage works as a methodological stance that enables us to focus on constant change rather than stability. This is to say that our analysis is sensitive to the premise that things - such as 'the self', the 'therapeutic' or the practice of 'self-tracking' - are constantly put together from diverse elements and are in a constant flux rather than fixed in place through notions of static 'essences' or 'cultures'. In relation to self-tracking as a proliferating practice of life-management, then, our aim is to show that there is no unequivocal way in which self-tracking 'is' a therapeutic practice of self-care. Rather, self-tracking 'becomes' therapeutic relationally, that is, in relation to the sociotechnical and political context in which it is practised.

\section{The fragmentary holism of self-tracking}

Self-tracking technologies are often implemented in everyday life and social imaginaries in manners that resonate with the therapeutic ethos of self-discovery and the pursuit of a self that is somehow 'whole'. Perhaps already due to the term self-tracking, the practice often becomes associated with the management of an undeniable uniqueness and wholeness of the person that an idea of the 'self' stands for. Yet, through general calls for 'self-knowledge through numbers', as the Quantified Self movement's popular slogan goes, people are encouraged to assemble self-knowledge through a wide variety of quantified data (Lupton 2016; Berg 2017). Different instances of and devices for self-tracking measure different and often very limited aspects of personal being - such as a step count or a heart rate. Therefore, self-tracking attracts very specific modes of knowing about personal existence. In being specific in this way, the activity already builds on a quite special notion of selfhood that steers away from looking at 'unique' life conditions and settings. In this section, we examine how self-tracking is framed as a therapeutic practice of making sense of the self, and how it is presented as a means for self-improvement. Through this analysis, we shed light on a contradictory tension internal to the dynamics of self-tracking, that we call fragmentary holism.

Our research materials show a plethora of ways by which technology developers narrate self-tracking devices' holistic capacities. Take, for example, Polar Electro Ltd., the manufacturer of popular fitness tracking wristbands that gather data on daily step counts and patterns of sleep. The company claims that monitoring by the wristband helps the person to become physically more active which, in turn, reduces health risks, increases personal well-being and improves general vitality. In the developer's promotional words, the device provides a 'complete' and 'truly holistic' picture of daily activity and 'highlights the importance of every movement'. ${ }^{2}$ Similarly, the Finnish wellness ring manufacturer Oura claims that '[w]ith Oura, you learn your optimal times to move, eat and take a break to 


\section{Harley Bergroth and Ilpo Helén}

get that restorative sleep'. ${ }^{3}$ The ring collects data on, for example, heart rates and nightly heart rate variability to inform users of their sleep quality. The developers call this a 'holistic method [...] built on years of experience in human performance and the study of circadian rhythms of the body'. Taking another example, HeartMath Inc. is a technology developer that focuses on stress reduction via the measurement of heart rate patterns. The company promotes devices such as the 'inner balance' sensor, and a method for constant monitoring of the changes of the heartbeat by claiming that such tracking leads one towards a state of coherence as long as one 'stays with it'. ${ }^{4}$ It further claims that measuring heart rate helps the person to 'incorporate the heart's intelligence into their day-to-day experience of life' and to 'become the best version [of oneself] more often'. As anthropologist Natascha Dow Schüll (2016) noted in her ethnographic study of health tech exhibitions in the US, the self-tracking industry's marketing constantly implies that the choices we make (in terms of attaining activity, good sleep, relaxation, etc.) reflect something crucial about who we really are.

In the above examples we see an adaptation of the therapeutic language of holistic self-understanding brought into the domain of digital self-tracking. The developers' claims often imply that the person may become something else as a whole through permanent tracking. For example, the individual is supposed to increase personal 'general vitality' and become 'physically more active' through step-focused activity tracking, or find 'coherence' and 'inner balance' via heartbeat data. Yet the tension between a holistic approach to personal life (referring to transformation of the individual as a whole) and 'fragmentary holism' (referring to linear optimisation of nuanced functions of the body) becomes evident in the marketing language. The developers paradoxically encourage a better holistic version of oneself (e.g. a more 'vital' or 'balanced' self) by focusing on an algorithmically predefined functionality (such as a step count or heart rate) or necessarily limited combinations of such functionalities. ${ }^{5}$ In addition, by encouraging people to become an improved version of themselves more often - for instance daily - the self-tracking imaginaries refer to an ongoing struggle of transformation. Thus, Polar, Oura and HeartMath end up presenting self-improvement as a linear and ongoing process (see Bode \& Kristensen, 2015) that is about constant monitoring and constant potentiality rather than about actuality.

Fragmentary holism highlights the idea that the 'self' is brought forth as an assemblage - disassembled and put back together through data - in self-tracking practices. First, the self consists of limited 'functions' or 'parameters' such as heartbeats or body movements, which are fragments of living that can be measured - and importantly, separated and combined - by means of self-tracking. In the case of an activity tracker, for example, a seemingly general picture of 'healthiness' or 'vitality' can be assembled by combining measurements on, for example, movement, heart rate and sleep quality. Second, through longitudinal measurement of any specific fragment or vital function, the self is enacted as a data assemblage. Several scholars point out the relational character of tracking as a practice that requires assembly work on separate yet entangled data points or data nodes (Ruckenstein, 2014; Day \& Lury, 2016; Schüll, 2016). This means 
that in order to make sense of and indicate progress or regress, any individual measurement (e.g. a step count) needs to be related to other similar measurements at different points in time.

We can illustrate the idea of the self as a data assemblage through the typical features of self-tracking software. Most self-tracking software visualises self-related data as graphs and charts that can be employed for self-development purposes. For example, the Polar Loop wristband gathers individual steps into longitudinal (daily and weekly) indicators of activity. In this way, the individual is disassembled into individual units of movement by the device's three-dimensional accelerometer, and re-assembled by the software. For example, depending on the device and software, a daily step assemblage on the application screen can take the form of a loop that gradually closes as one gathers steps and nears the daily goal, or a bar that fills up accordingly. A weekly activity assemblage may be visually presented e.g. as a bar chart. Similar logic pertains to measurements of heart rate, sleep, etc.; individual beats or movements are assembled into daily/nightly stats, which are then further assembled into longitudinal graphs. It is also typical that a simple number indicates the tracked self in a moral sense. For example, a self-tracking application may make complimentary claims about the person based on their total number of daily or weekly steps, or offer an 'efficiency percentage' for daily/weekly activity or sleep. Achieving high numbers or hitting 100 percent are then often virtually rewarded with animations, colour codes, trophies, etc. Here individual measurements or measurement combinations are algorithmically assembled into an evaluation. It is typical that such 'holistic' indicators of the self can again be disassembled into smaller-scale information in order to get more nuanced data on the specific function, for instance, to see the distribution of steps on a daily or hourly basis. However, in everyday use, to see the 'importance of every movement', as Polar mentions as a goal, is to ultimately trust the algorithm that assembles a personal evaluation. However, completion of a single evaluative checkpoint (e.g. a single day) is never really a completion at all. For example, in the Polar Loop activity tracking software, the person is implicitly encouraged to reach $100 \%$ activity (i.e. enough movement) daily. However, the weekly visualisation of one's activity shows a 'daily goal completion average', which groups together daily evaluations and offers a different number, the average.

Considering the self as a data assemblage also means that individual measurements of different functions could or should also ideally be related to each other. For example, according to Polar, when combined with a heart rate monitor, the activity wristband can become an even more precise indicator of 'activity', as it can then offer more accurate quantification-based insight on the intensity of activity conducted, calories burned, etc. It can be argued that the more elements the assemblage has, the more 'holistic' the image of the self it paints. However, the more elements there are, the more data there are on separate functions that can, or need to, be tied together and related to other measurement data.

The point here is that as the tracked self is enacted as a data assemblage, selftracking as a way of working upon the self always feeds back to the user through its limitations. Data could always be more complete and more saturated through 


\section{Harley Bergroth and Ilpo Helén}

relations, references and repetition. Therefore, the practice is in principle opposed to the idea of a 'holistic' understanding of the self. Of course, we do not deny that self-trackers often pay attention to qualitative data - for example, their experiences and sensations - in order to make deeper sense of the quantitative data (see e.g. Ruckenstein, 2014; Lupton, 2016). However, while we grant that people may well experience the gaining of insights into their lives by contextualising the data, we think that holistic humanism and reflexive hermeneutics of the self often tend to be eclipsed or even be made redundant by the technical affordances of self-tracking: the precision of measurement of a specific function or activity at a specific point in time. Polar, for example, directly presents the process of 'becoming a more active person' as a project in which the person becomes conscious of her/his step-based physical movement within a day in specific ways and adjusts her/his behaviour to modify these specific modes of action. Such awareness and a trajectory of personal action are induced by the measurement because physical movement (or lack of it) is what the devices and algorithms recognise, react to and respond to by producing data that then provide feedback for the user.

To sum up, from the viewpoint of assemblages of control at work in selftracking, individuals become increasingly conceived of as compounds of functions, parameters, capacities and resources to be regulated and operated upon. Such control as a mode of governing people and their lives sits uneasily with the holism that nevertheless surfaces as an emic emphasis in the imaginaries of the capacities of self-tracking. For holistic therapeutic practices, the individual person and her/his 'unique' life, experiences and aspirations are the focus of practices to govern and modify people, whereas control and dividualisation reach beyond and below the personal by means of the collection and aggregation of masses of data. We claim that while many therapeutic practices are concerned with encouraging people to discover their unique modes of action for self-improvement, digital selftracking today becomes functional by focusing on self-control and by improving modes of action already predetermined in the devices' code. In what follows, we will take a closer look at everyday experiences of self-tracking and discuss these experiences as reflective of anticipatory regimes of action.

\section{Self-tracking as a therapeutic regime of anticipation}

So far we have seen that self-tracking is often presented as a holistic means of self-inspection despite its tendency to disassemble the self into (longitudinal collections of) functions and variables, which encourages further selfinspection rather than 'holistic' self-knowledge. In this section, we focus on the ways in which self-tracking assemblages are narrated to facilitate the idea and ongoing pursuit of a good - or, rather, better - life. We thus show how the data assemblages gathered in self-tracking practices unfold into therapeutic regimes of action in everyday lives.

When people speak of their self-tracking practices they usually say that selftracking makes aspects of the self (activity levels, sleep quality, etc.) 'more real'. Sari, a woman in her late forties, speaks about how the Polar Loop device enables 
her to convince herself that she is 'really doing something' in the sense of daily activity. Specifically, she has tried to fight against a risk of type 2 diabetes that has been brought to her attention by medical professionals. When Timo (male, 26) talks about tracking daily movement and calorie consumption, he readily recognises the very limited employability of individual measurements and says that he does not necessarily check on the measurements on a daily but rather on a weekly basis. Despite this, he points out that the accumulation of data reveals patterns and creates a very tangible or 'real' history, evident in the repository of numbers and graphs. Through this timeline he can then reflect on and analyse 'how things have been going' in his life in a more general sense. It can be argued that self-tracking usually makes sense to people as a practice of acquiring knowledge about developments in terms of specific functions, which is also thought to reveal a bigger picture of one's own life through futures and histories of potential health, physical fitness and wellbeing.

However, despite such experiences, what is characteristic of interviewees' narratives is the pervasive tension between becoming a self-informed subject and constantly pushing the boundaries of self-knowledge further. In many cases, then, self-tracking in practice becomes anticipatory (see e.g. Adams et al., 2009) rather than evidentiary. Many interviewees acknowledge that self-tracking may provide quite a lot of self-awareness and promote joy and delightful moments when the self is actualised as 'active', for example, by reaching 10,000 steps or by producing a good sleep score daily or, even better, consistently, but they also reflect that it may easily turn into a repetitive practice, the main attraction of which is to predict and control. As the 'tracks' always lead on, self-tracking can spark ever further interest in the self, which makes the idea of the self as an experience of something 'whole' quite questionable. For example, Veli, a 28 -year-old male, reflects that it is really 'quite silly' to engage in sleep tracking because in a sense one already knows how one sleeps. Nevertheless, he self-tracks because the data logs 'provide motivation for improvement'; that is, the logs and linear graphs psychologically move him to develop himself. This highlights the tendency of self-tracking to produce a processual regime of action in terms of datafied self-mapping.

It is our claim that through fragmentary holism, the purpose of self-tracking often becomes the process of self-assembly itself, as a sense of self-control is pursued. Relating to dividualisation, it seems that self-tracking as a therapeutic assemblage engenders a mode of action that enables not holistic self-awareness per se but rather an information-deprived relation to the self on pre-established scales. In other words, the self becomes highlighted as potential. This relates to how, in the context of border security, Amoore (2011: 28) speaks of algorithmically forged 'data derivatives' that do not centre on 'who we are, not even on what our data says about us, but what can be imagined and inferred about who we might be'. Self-tracking data derivatives are another example of how improvement is linked with attempted control of potential futures and threats.

In terms of specific threats, our analysis of the promotional material points out that self-tracking devices are often framed as quasimedical devices that are supposed to lead individuals towards health, longevity and regeneration. As such, 


\section{Harley Bergroth and Ilpo Helén}

they both answer to and produce what anthropologist Joseph Dumit (2012; see also Schüll, 2016) calls a 'double insecurity' that the medical industry enacts: not knowing whether one is already ill and never knowing enough about illness prevention. The person is thus always potentially ill. Perhaps the clearest example of this in our interviews comes from Sakari, a 50-year-old male who is a very active self-tracker and describes himself as a scientist of his own life (cf. Heyen, 2016). For example, he makes an effort to track his weight and relaxation (via heart rate) daily, his blood pressure regularly once a year, and occasionally he goes to a nearby laboratory to gain data on various biomarkers of his choice. All this provides him with 'more data' in his ever-expanding database of himself. In addition to quantitative data, he also carefully notes any events that may be symptoms of illness. (One example that he provided us with is ' 12 th March, nosebleed, short duration, left nostril'.) He describes self-tracking as 'at its best a very therapeutic practice' in the sense of producing a 'peace of mind' and feelings of 'self-confidence'. However, he also states that a central product of self-tracking is the feeling of 'panic' or 'terror', especially when one sees results that deviate from normal reference values and seem unexplained to him. When asked about whether self-tracking has served his well-being or not, Sakari said that a sense of control provides him with a really 'healthy feeling'. Explaining the negative side of tracking in more detail, Sakari specified his ideas on control:

It is really about an experience of control... because you cannot really control life, but you can feel that you are in control... and the negative comes from the kind of terror, especially in the face of [measurement] results that are somehow... unexplained [...] and it seems hard for me to grasp what could be negative about [self-tracking]... like if you think that people get all hypochondriac and they increasingly go to the doctor, well I think that's a good thing in a way. Because this is preventive health care, and it's better that you go early [to the doctor] to check up on some fine nuance, than going when you're already sick.

For Sakari, self-tracking seems to become functional as self-control and an ongoing struggle against illness. However, as his life and body are now spatially and temporally divided into ever-expanding data sets by and through self-tracking technologies, it is also quite likely that he will encounter situations in which the feeling of a loss of control, in the form of unexplained data, becomes tangible. He explicitly connects such scenarios with emotions of terror. Here we can see self-tracking as a therapeutic assemblage at work: self-tracking becomes therapeutic as a practice of continuous and preventive health-related control through the dividualising and fragmentary logic of the system. This fragmentation and production of self-related data derivatives mean that a sense of full control often remains elusive. In Sakari's case, this is implied in the curious sense that selftracking seems both a vitally important and therapeutic task, but also, in the long run, a battle one cannot win, because 'you cannot really control life'. Thus, the therapeutic assemblage of self-tracking produces its own purpose by opening up 
the self and the body as potential and as an object of continuous control. The self becomes a data assemblage that is not, in fact, approached as a whole individual but as dividualised into trajectories of functions, traits and biomarkers, through monitoring of which a sense of control may be pursued.

As control of potential, self-tracking may be thought of as a constant struggle against the 'deviant' within (Bode \& Kristensen, 2015). While Sakari wages war on various fronts against ill health, in another example we see the struggle against the disorder and social ill of 'laziness' or 'idleness', familiar from mainstream wellness- and efficiency-related activation discourses. ${ }^{6}$ Aino is a 39 -yearold female, a mother of three children who works in an executive role. She has a background in competitive sports so she has long been familiar with heart rate monitors and other gadgets by which one can optimise physical performance via metrics. For Aino, such techniques have now become a part of coping with the demands of working life. She says that an activity wristband motivates her to move more, and she feels that if she did not exercise she 'would not have the energy to cope with [the] damn tough demands' of a stressful job. Here, as with Sakari, the way in which self-tracking comes to serve as a useful technology of the self (Foucault, 1988) is by control of vital potential, although in this case self-tracking is more explicitly connected to the maintenance of one's productive energy. Aino speaks about self-tracking as an ongoing process of avoidance of the lazy self always lurking in the shadows. She says that the whole point is to 'give yourself a kick up the ass' and 'avoid the days when [my] activity is basically zero'. She wants to avoid inactivity, which she explicitly associates with 'laziness'. In a quote Aino reveals how the device cooperates daily in establishing a sense of self-control:

Of course it was nice when in the summer I went golfing and I got a huge amount of steps... of course it was nice [smiles widely], like WOW, so many steps. But in normal life it is enough for me that the wristband vibrates [haptic vibration, a signal of achieving 10,000 steps] at some point of the day, that I've been active.

It goes without saying that a life with an activity tracking wristband that counts steps and measures sleep via movement of the body may enable different modes and patterns of self-control than a life with a weighing scale or with access to high-end laboratory testing. However, the logic of dividualisation in any case frames one's relation to the self as a relation of control, because the production of the self as data assemblage highlights the self as potential. This is evident in Sakari's and Aino's cases. Sakari is potentially always in ill health, even (or perhaps especially) when there are no obvious indications of illness (see Dumit, 2012). The complex assemblages of technical devices, laboratories and biographical notes seemingly enable the person to control this insecurity, although such assemblages also produce the self as an object of 'tinkering' (Pols, 2010) with fine-grained nuances. In a similar manner, Aino is potentially always 'lazy', since every day is another struggle against 'laziness' and 'low energy', which in her 
case is determined primarily via step count. The process of self-management manifests itself as permanent avoidance of the illness of 'zero' activity.

We suggest that while everyday proactive self-tracking is often narrated in terms of the typical therapeutic parlance of self-discovery, self-exploration or self-improvement, interview narratives show that dividualisation and fragmentation of the self are the primary characteristics of self-tracking. Although it may be said that self-tracking enables a process of self-discovery through the longitudinal measurement of fragmented vital functions or activities, in functioning it also creates interests that tie individuals into these sociotechnical regimes of control. The functions of the data as therapeutic life management ultimately become anticipatory rather than evidentiary, as any individual event of tracking does not so much generate self-knowledge as position the self in a continuum of measurements through which future (and past) selves can be imagined and potentially realised. Self-tracking thus persistently produces knowledge-craving subjects whom it supposedly serves, and the self as a 'whole' remains persistently unattainable. Self-tracking assemblages, then, present vivid instantiations of control through anticipation because, as we have seen, from marketing rhetoric to everyday experiences these technologies reveal the self as process and as potential, as something to be acted upon consistently in order to actualise a better life. Inasmuch as self-tracking assemblages are articulated as producing knowledge of something 'real' about the self, they create a need to know by dividing a complex whole of life into trajectories and functions that extend indefinitely. This is fragmentary holism in action.

\section{Therapeutic self-assembly and the politics of self-control}

Deleuze (1995b: 179) wrote that whereas life in disciplinary societies is characterised by completions and new beginnings, like trajectories from families to schools, and from schools to work, 'in control societies you never finish anything'. In this chapter, we have shown how self-tracking as a technology of the self often drives fragmentation rather than unity, and anticipation rather than knowledge. Selftracking enacts the self, through a focus on linear scales, as an ongoing process and potentiality rather than as something to be found as a whole and coherent. An important consequence is that self-tracking assemblages bring to everyday life a modality of being characterised by perpetual alteration. From the consumer perspective, the therapeutic ethos of holism and stability thus appears appealing and welcome as it promises control, yet it is ultimately futile within the assemblages that produce their raison d'être through producing a metastable existence - an existence that is constantly subject to change. Acting to manage this metastability associates self-tracking as a therapeutic regime with the anticipatory control and regulation of potentiality in political regimes. In the last section of our chapter, we probe briefly this political aspect of self-tracking as control.

Our analysis of self-trackers' narratives brought up struggles against ill health and laziness, both of which are identifiable as long-running trajectories of struggle in Western ethics. Considering these struggles further along with Deleuze's 
ideas of control society we may connect them with sociotechnical visions and programmes of 'perpetual education' and 'personalised medicine' which rely on processes of dividualisation. If we consider self-tracking, on a general level, as perpetual education - resonant with what Fotopoulou and O'Riordan (2017) call 'biopedagogy' - we can connect it with social programmes of activation. In critical social policy literature and in public discourse, 'activation' has typically referred to a variety of local and international policies, hugely influential across OECD countries, through which the unemployed or the 'excluded' have been made responsible for managing their labour power, working abilities and personal life in general (Eversberg, 2015: 173; Clarke, 2005: 448). Yet, as a programme or an assemblage, activation is itself contingent; it takes shape in relation to neoliberal market rationale and the logics of restructuring of the welfare state, through which the state and public powers seemingly withdraw from securing individual lives and from interfering with them. Furthermore, 'activation' may work as an umbrella term that covers a variety of traits and qualities such as, for example, education, the utilisation of prior work experience, health, well-being, mental awareness or aesthetic appearance. All of these are closely intertwined with the ability to self-manage and maintain the social and economic utility that is, labour capacity and productivity - of one's own body. In this context, the point of self-tracking is to educate people not on their daily step counts or heart rates during sleep per se, but mainly on caring for and managing personal 'vitality' by themselves in order to reduce the 'deadweight' in the productive system (see Eversberg, 2015). Activation is thus a programme that is actualised through mundane technologies far beyond its rhetorical target population, that is, the unemployed, the precariat and the 'excluded' who are typically not the target group for self-tracking devices. We see how activation becomes actual with Aino, who worries about - and finds therapeutic functions in - managing her energies and capacities, that is, her labour power, through a struggle against laziness in a demanding and stressful work environment. In Virve Peteri's chapter in this book, we see spatial arrangements of the office space as a new mode of activation and mobilisation of workers and their labour power within organisations; in this chapter, we see parallel strategies of activation with self-tracking technologies in everyday life and outside organisational contexts.

Self-tracking as an assemblage of control also has an affinity, and perhaps a more concrete one, with a major imaginary of future healthcare envisaged as 'personalised, predictive, preventive, and participatory' (Hood \& Friend, 2011). The advocates of 'personalised medicine' conceive of it as being embedded in advanced biomedicine like genomics and stem cell technology and claim that it will improve clinical care and shift the emphasis of health care to prevention with the help of more precise and patient-centred medical knowledge (for an overview, see Tutton, 2014). In addition, personalised medicine is expected to considerably reduce the costs of healthcare. Over the past few years, visions of personalised medicine have re-focused on the collection and appropriation of masses of health-related personal data (Prainsack, 2017; Ruckenstein \& Schüll, 2017). According to the promoters, data-driven medicine would enable anticipatory 
health monitoring and preventive interventions, as well as medication and other therapies, targeted far more precisely at specific risk groups or individuals (e.g. NAS, 2011; Hood \& Friend, 2011).

Self-tracking works in congruence with the data-driven personalised medicine that is expected to revolutionise modern medicine, health policy and society (NAS, 2011; Hood \& Friend, 2011; Mayer-Schönberger \& Cukier, 2013; Pentland, 2013; Topol, 2015). This congruence builds through the logics of control and dividualisation, both of which are embedded in massive data sets and have a focus on individual parameters and malleable patterns (see Sharon, 2017). Personalised medicine is often seen as a frame for preventive lifestyle and proactive medical interventions, supported by perpetual self-monitoring and control. As seen in our examples, many companies promoting self-tracking - as well as self-trackers themselves, such as Sakari - focus on the measurement of healthrelated parameters of vital functions and behaviour and narrate self-optimisation by digital tracking devices in a manner similar to preventive healthcare.

Personalised medicine is expected to have effects across populations, societies and even globally. The dividualisation taking place in such practices also lays the ground for the pervasiveness of 'therapy cultures' as personal lives are perpetually in need of preventive interventions: for example, always rather potentially ill (metastable) than healthy organisms (stable). Personalised medicine is thus another political programme that lays the ground for the sociotechnical instantiations and alterations that are shaping current therapy cultures towards a focus on ongoing self-control and metastability rather than healing, wholeness and stability.

\section{Conclusion}

In this chapter, we argue that while self-tracking can be theorised in terms of its connections to the general therapeutic ethos of self-discovery and self-improvement, it is a data-driven practice of dividualisation. As such, it creates regimes of action that build on the idea of perpetual self-assembly and which thus fit uneasily with any overarching characterisation of 'therapeutic cultures'. Instead of holistic and reflexive self-inspection we often see the fragmentation of the individuals and their lives into 'functions' and 'qualities' presented by graphs and charts, and in ways that focus on the self as a process that should be worked upon consistently. So, in terms of how these technologies come to serve life, they serve not as holistic actualisation of the self but as a means of ongoing control and management of potential. As life-management techniques, these technologies have a tendency to actively produce the kinds of regimes of perpetual action that they promise to dissolve. Furthermore, self-tracking as a technology of the self exemplifies how current therapeutic assemblages can also intertwine with political programmes and discourses such as citizen activation and personalised medicine. We see selftracking as to some degree pertaining to the emergence of societies of control as sketched by Deleuze, especially through a focus on increasing complexity and persistent incompleteness, which both attract endless monitoring. 


\section{Acknowledgements}

The authors wish to thank the Academy of Finland (grant number 292408, grant number 313703, grant number 289004) and Kone Foundation (Crossing Borders for Health and Well-being) for supporting this work.

\section{Notes}

1 Mika Pantzar (2012: 133) has noted that when Finnish technology developers such as Polar Electro tried to introduce and sell heart rate monitors to American consumers at the end of the 20th century, they faced resistance as it was thought unclear why the average consumer should need one.

$2 \mathrm{https}: / /$ www.polar.com/us-en/about_polar/press_room/polar_launches_polar_loop and https://support.polar.com/fi/support/the_what_and_how_of_polar_24_7_activity tracking?product_id $=64271 \&$ category $=$ faqs $[$ [accessed on March 23, $\overline{0}$ - $18 \overline{\text { ] }}$.

$3 \mathrm{https} / / /$ ouraring.com/ [accessed on March 28, 2018, italic as in original source].

$4 \mathrm{https}: / /$ store.heartmath.com/innerbalance [accessed on March 28, 2018].

5 For example, a measurement of sleep quality may be based on a simple parameter, such as movement, or it may be based on a combination of parameters, such as movement data, heart rate data and data on breathing rhythms.

6 For example, these discourses now frame sitting as a public health threat (Peteri, 2017) and manifest themselves in various campaigns in workplaces encouraging people to be physically active during workdays.

\section{References}

Adams, V., Murphy, M. \& Clarke, A. 2009. Anticipation: Technoscience, life, affect, temporality. Subjectivity 28, 246-265.

Amoore, L. 2011. Data derivatives: On the emergence of a security risk calculus for our times. Theory, Culture \& Society 28:6, 24-43.

Beer, D. 2016. Metric Power. London: Palgrave MacMillan.

Bennett, J. 2010. Vibrant Matter: A Political Ecology of Things. Durham: Duke University Press.

Berg, M. 2017. Making sense with sensors: Self-tracking and the temporality of wellbeing. Digital Health 3, 1-11.

Bode, M. \& Kristensen, D. B. 2015. The digital doppelgänger within: A study on selftracking and the quantified self movement. In Assembling Consumption: Researching Actors, Networks and Markets, edited by R. Canniford \& D. Bajde. London: Routledge, $119-135$.

Clarke, J. 2005. New labour's citizens: Activated, empowered, responsibilized, abandoned? Critical Social Policy 25:4, 447-463.

Crawford, K., Lingel, J. \& Karppi, T. 2015. Our metrics, ourselves: A hundred years of self-tracking from the weight scale to the wrist wearable device. European Journal of Cultural Studies 18:4-5, 479-496.

Day, S. \& Lury, C. 2016. Biosensing: Tracking persons. In Quantified: Biosensing Technologies in Everyday Life, edited by D. Nafus. Cambridge: MIT Press, 43-66.

Deleuze, G. 1995a. 'Control and becoming'. In Negotiations, 1972-1990. New York: Columbia University Press, 169-176.

Deleuze, G. 1995b. 'Postscript on control societies'. In Negotiations, 1972-1990. New York: Columbia University Press, 177-182. 


\section{Harley Bergroth and Ilpo Helén}

Deleuze, G. \& Guattari, F. 1987. A Thousand Plateaus. Minneapolis: University of Minnesota Press.

Dumit, J. 2012. Drugs for Life: How Pharmaceutical Companies Define Our Health. Durham \& London: Duke University Press.

Eversberg, D. 2015. Beyond individualisation: The German 'activation toolbox'. Critical Social Policy 36:2, 167-186.

Fotopoulou, A. \& O'Riordan, K. 2017. Training to self-care: Fitness tracking, biopedagogy and the healthy consumer. Health Sociology Review 26:1, 54-68.

Foucault, M. 1988. Technologies of the self. In Technologies of the Self: A Seminar with Michel Foucault, edited by M. Gutman \& P. Hutton. London: Tavistock, 16-49.

Foucault, M. 1993. About the beginnings of the hermeneutics of the self. Political Theory $21,198-227$.

Heyen, N. B. 2016. Self-tracking as knowledge production: Quantified self between prosumption and citizen science. In Lifelogging, edited by S. Selke. Wiesbaden: Springer VS.

Hood, L. \& Friend, S. 2011. Predictive, personalized, preventive, participatory (P4) cancer medicine. Nature Reviews Clinical Oncology 8:3, 184-187.

Illouz, E. 2008. Saving the Modern Soul: Therapy, Emotions, and the Culture of Self-Help. Berkeley: University of California Press.

Latour, B. 2005. Reassembling the Social: An Introduction to Actor-Network Theory. Oxford: Oxford University Press.

Lomborg, S. \& Frandsen, K. 2016. Self-tracking as communication. Information, Communication \& Society 19:7, 1015-1027.

Lupton, D. 2013. Understanding the human machine. IEEE Technology and Society Magazine 32:4, 25-30.

Lupton, D. 2016. The Quantified Self: A Sociology of Self-Tracking. Cambridge: Polity Press.

Madsen, O. J. 2014. The Therapeutic Turn: How Psychology Altered Western Culture. London \& New York: Routledge.

Madsen, O. J. 2015. Optimizing the Self: Social Representations of Self-Help. London: Routledge.

Marcus, George E. \& Saka, E. 2006. Assemblage. Theory, Culture \& Society 23:2-3, 101-109.

Mayer-Schönberger, V. \& Cukier, K. 2013. Big Data: A Revolution That Will Transform How We Live, Work and Think. London: John Murray.

McGee, M. 2005. Self-Help Inc. Makeover Culture in American Life. Oxford: Oxford University Press.

Mol, A. 2002. The Body Multiple: Ontology in Medical Practice. Durham: Duke University Press.

Moskowitz, Eva. 2001. In Therapy We Trust: America's Obsession with Self-Fulfillment. Baltimore: The Johns Hopkins University Press.

National Academy of Sciences [NAS]. 2011. Toward Precision Medicine. Washington, DC: The National Academies Press.

Pantzar, M. 2012. Arjen uudet kartastot ja rytmit: Big data ja small data. In Arjen kartat ja rytmit, edited by M. Lammi, M. Pantzar \& T. Koivunen. Kuluttajatutkimuksen vuosikirja 2012. Helsinki: Kuluttajatutkimuskeskus, 128-151.

Pentland, A. 2013. The data-driven society. Scientific American 309:4, 78-83.

Peteri, V. 2017. Tuolin imu: kansanterveys, kansallinen kilpailukyky ja istumisen vaarat. Kulttuurintutkimus 34:1, 15-27. 
Pols, J. 2010. Telecare: What patients care about. In Care in Practice. On Tinkering in Clinics, Homes and Farms, edited by A. Mol, I. Moser \& J. Pols. Bielefeld: Transcript Verlag, 171-194.

Prainsack, B. 2017. Personalized Medicine: Empowered Patients in the 21st Century. New York, NY: New York University Press.

Rimke, H. 2000. Governing citizens through self-help literature. Cultural Studies 14:1, 61-78.

Ruckenstein, M. 2014. Visualized and interacted life: Personal analytics and engagements with data doubles. Societies 4:1, 68-84.

Ruckenstein, M. \& Schüll, N. D. 2017. The datafication of health. Annual Review of Anthropology 46, 261-278.

Schüll, N. D. 2016. Data for life: Wearable technology and the design of self-care. BioSocieties 11:3, 317-333.

Sharon, T. 2017. Self-tracking for health and the quantified self: Re-articulating autonomy, solidarity and authenticity in the age of personalized healthcare. Philosophy \& Technology 30:1, 93-121.

Topol, E. 2012. The Creative Destruction of Medicine. New York: Basic Books.

Topol, E. 2015. The Patient Will See You Now: The Future of Medicine Is in Your Hands. New York: Basic Books.

Tutton, R. 2014. Genomics and the Reimagining of Personalized Medicine. Surrey: Ashgate. 